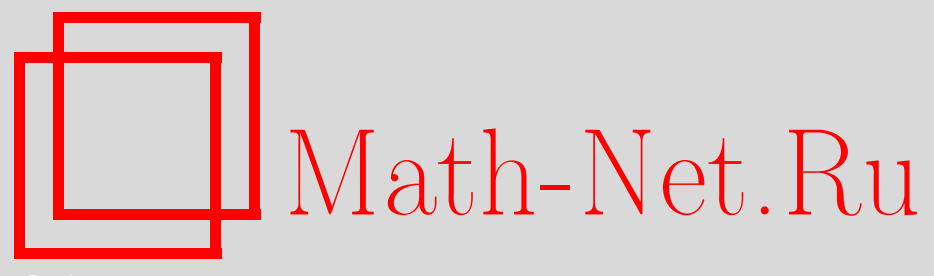

Д. Б. Рохлин, О существовании эквивалентной супермартингальной плотности для разветвленно-выпуклого семейства случайных процессов, Матем. заметки, 2010, том 87, выпуск 4, 594-603

DOI: https://doi.org/10.4213/mzm4151

Использование Общероссийского математического портала Math-Net.Ru подразумевает, что вы прочитали и согласны с пользовательским соглашением http://www . mathnet.ru/rus/agreement

Параметры загрузки:

IP : 54.89 .56 .158

26 апреля 2023 г., 15:58:34

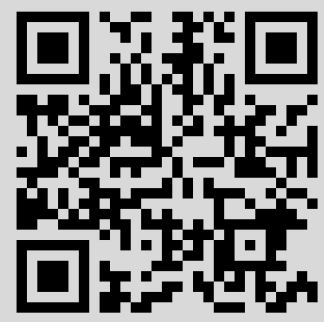


Том 87 выпуск 4 апрель 2010

УДК 519.216 .8

\section{О существовании эквивалентной супермартингальной плотности для разветвленно-выпуклого семейства случайных процессов}

\section{Д. Б. Рохлин}

Доказано, что разветвленно-выпуклое семейство $\mathbb{W}$ неотрицательных случайных процессов обладает эквивалентной супермартингальной плотностью, если и только если множество $H$ неотрицательных случайных величин, мажорируемых значениями элементов $\mathbb{W}$ в фиксированные моменты времени, ограничено по вероятности. Условиям данной теоремы удовлетворяет модель рынка ценных бумаг с произвольным числом основных рисковых активов, представляющая собой множество $\mathbb{W}(\mathbb{S})$ неотрицательных стохастических интегралов по конечным наборам семимартингалов из произвольного индексированного семейства $\mathbb{S}$.

Библиография: 18 названий.

1. Основные результаты. Рассмотрим вероятностное пространство $(\Omega, \mathscr{F}, \mathrm{P})$, наделенное фильтрацией $\left(\mathscr{F}_{t}\right)_{t \in \mathbb{R}_{+}}, \mathbb{R}_{+}=[0, \infty)$ (т.е. неубывающим семейством $\sigma$-алгебр $\left.\mathscr{F}_{t} \subset \mathscr{F}\right)$, удовлетворяющей обычным условиям непрерывности справа и полноты [1]. Предполагается, что $\mathscr{F}=\sigma\left(\bigcup_{t \geqslant 0} \mathscr{F}_{t}\right)$ и $\sigma$-алгебра $\mathscr{F} 0$ тривиальна с точностью до Р-нулевых множеств. Все рассматриваемые далее случайные процессы считаются согласованными с фильтрацией $\left(\mathscr{F}_{t}\right)_{t \in \mathbb{R}_{+}}$. Пусть $\mathbb{D}-$ множество случайных процессов, траектории которых непрерывны справа и имеют конечные пределы слева Р-п.н.

Обозначим через $L^{0}=L^{0}(\Omega, \mathscr{F}, \mathrm{P})$ пространство классов эквивалентности $\mathscr{F}$-измеримых случайных величин. Наделим $L^{0}$ топологией, порожденной метрикой

$$
\rho(f, g)=\mathrm{E} \frac{|f-g|}{1+|f-g|} .
$$

Сходимость в такой топологии совпадает со сходимостью по вероятности, а ограниченность - с ограниченностью по вероятности. Напомним, что множество $F \in L^{0}$ называется ограниченным по вероятности, если для любого $\varepsilon>0$ существует $M>0$ такое, что $\mathrm{P}(|f| \geqslant M)<\varepsilon, f \in F$. Через $L_{+}^{0}$ обозначим конус неотрицательных элементов $L^{0}$.

Работа выполнена при поддержке Российского фонда фундаментальных исследований (грант № 07-01-00520).

(C) Д. Б. Рохлин, 2010 
Следуя [2], назовем семейство $\mathbb{W} \subset \mathbb{D}$ неотрицательных случайных процессов разветвленно-выпуклым (fork-convex), если для любых элементов $X^{i} \in \mathbb{W}, i=1,2,3$, где $X^{2}>0, X^{3}>0$, любого $s \in \mathbb{R}_{+}$и любого $h_{s} \in L_{+}^{0}\left(\mathscr{F}_{s}\right), h_{s} \leqslant 1$, процесс

$$
X_{t}=X_{t}^{1} I_{[0, s)}(t)+X_{s}^{1}\left(h_{s} \frac{X_{t}^{2}}{X_{s}^{2}}+\left(1-h_{s}\right) \frac{X_{t}^{3}}{X_{s}^{3}}\right) I_{[s, \infty)}(t)
$$

принадлежит $\mathbb{W}$. Легко видеть, что если $X_{0}=1$ для всех $X \in \mathbb{W}$, то разветвленно-выпуклое множество $\mathbb{W}$ является выпуклым. Если же $1 \in \mathbb{W}$, то $\mathbb{W}$ инвариантно относительно остановки в фиксированные моменты времени, т.е. процесс $X_{t}^{T}=X_{T \wedge t}$ принадлежит $\mathbb{W}$ вместе с $X$. Здесь $a \wedge b=\min \{a, b\}$.

Напомним, что случайный процесс $Y$ называется супермартингалом (относителъно меры Р), если случайные величины $Y_{t}$ являются Р-интегрируемыми и условное математическое ожидание $\mathrm{E}\left(Y_{t} \mid \mathscr{F}_{s}\right)$ не превосходит $Y_{s}$ п.н. при $s<t$. Случайный процесс $Z \in \mathbb{D}$, удовлетворяющий условиям $Z_{0}=1, Z_{t}>0, t>0$, и

$$
Z_{\infty}=\lim _{t \rightarrow \infty} Z_{t}>0 \text { п.н., }
$$

назовем эквивалентной супермартингальной плотностью для $\mathbb{W}$, если процесс $X Z$ является Р-супермартингалом для любого $X \in \mathbb{W}$. Аналогичные объекты вводились, например, в работах [3]-[5]. Называя "эквивалентными" плотности с условием $Z_{\infty}>0$, мы следуем терминологии [6].

Введем множество $H$ неотрицательных случайных величин, мажорируемых значениями элементов $\mathbb{W}$ в фиксированные моменты времени:

$$
H=\left\{y \in L_{+}^{0}: y \leqslant X_{T} \text { для некоторых } X \in \mathbb{W}, T \geqslant 0\right\} .
$$

Основной результат настоящей работы состоит в следующем.

ТЕОРема 1. Пусть $\mathbb{W}$ - разветвленно-выпуклое семейство случайных прочессов, содержащее 1, и пусть $X_{0}=1$ для всех $X \in \mathbb{W}$. Тогда следующие условия эквивалентны:

(i) множество (1.2) ограничено по вероятности;

(ii) существует эквивалентная супермартингальная плотность для $\mathbb{W}$.

Данный результат имеет ясную интерпретацию в рамках математической теории арбитража [7]. Именно, пусть имеется произвольное индексированное семейство $\mathbb{S}=\left(S^{i}\right)_{i \in J}$ семимартингалов $S^{i} \in \mathbb{D}$ (определения семимартингала и векторного стохастического интеграла см. в [1]). Через $L(\mathbb{S})$ обозначим множество, элементами которого являются семейства $\Gamma=\left(\gamma^{i}\right)_{i \in J}$ предсказуемых случайных процессов, удовлетворяющих следующим условиям:

(a) $\gamma_{i}=0$ для $i \in J \backslash I$, где $I$ - некоторое конечное множество (зависящее от $\Gamma$ );

(b) определен векторный стохастический интеграл $\left(\gamma^{i}\right)_{i \in I}$ по $\left(S^{i}\right)_{i \in I}$.

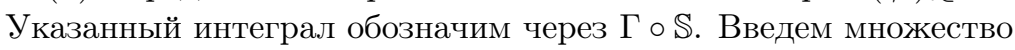

$$
\mathbb{W}(\mathbb{S})=\{X \in \mathbb{D}: X=1+\Gamma \circ \mathbb{S} \geqslant 0, \Gamma \in L(\mathbb{S})\}
$$

Данную конструкцию можно рассматривать как модель рынка с произвольным числом основных рисковых активов. Процесс $S^{i}$ описывает цену $i$-го рискового актива, $\gamma^{i}$ - количество указанного актива в портфеле инвестора, $X$ - капитал допустимой инвестиционной стратегии. Следуя [6], будем говорить, что в рассматриваемой 
модели рынка выполнено условие отсутствия неоганиченной прибыли с ограниченным риском (NUPBR: No Unbounded Profit with Bounded Risk), если множество

$$
\left\{y \in L_{+}^{0}: y \leqslant X_{T} \text { для некоторых } X \in \mathbb{W}(\mathbb{S}), T \geqslant 0\right\}
$$

ограничено по вероятности.

TЕОРема 2. Для выполнения условия NUPBR необходимо и достаточно существования эквивалентной супермартингалъной плотности для $\mathbb{W}(\mathbb{S})$.

В случае конечного числа активов (т.е. конечного множества $J$ ) данный результат содержится в работе [6], где используется тонкая техника стохастического исчисления. Методы настоящей работы позволяют дать неожиданно короткое доказательство теоремы 2 (для произвольного $J$ ), основанное лишь на стандартных теоремах функционального анализа и теории мартингалов. Данный результат можно рассматривать так же, как критерий отсутствия асимптотического арбитража первого рода на большом финансовом рынке [8], [9], заданном на фиксированном вероятностном пространстве (как в работе [10]).

Полезно сопоставить теорему 2 с более известной формой первой фундаментальной теоремы финансовой математики [11], [12]. Рассмотрим рынок с конечным числом активов ( $J$ конечно). Будем говорить, что выполнено условие отсутствия арбитража (NA: No Arbitrage), если из условий $X \in \mathbb{W}(\mathbb{S}), X_{\infty} \geqslant 1$ п.н. (при условии, что данный предел существует) вытекает, что $X_{\infty}=1$ п.н. Вероятностная мера $Q$ называется эквивалентной супермартингальной мерой для $\mathbb{W}(\mathbb{S})$, если $Q$ и $\mathrm{P}$ обладают одинаковым запасом нулевых множеств и все процессы $X \in \mathbb{W}(\mathbb{S})$ являются супермартингалами относительно Q.

Следующий результат [6], представляет собой удобную переформулировку первой фундаментальной теоремы [11], [12].

ТеОРема 3. В модели рынка с конечным числом активов для выполнения условий NA и NUPBR необходимо и достаточно существования эквивалентной супермартингальной меры для $\mathbb{W}(\mathbb{S})$.

Подчеркнем, что предлагаемый подход не приводит к столь же короткому доказательству теоремы 3. Кроме того, данная теорема не допускает непосредственного обобщения на модели с бесконечным числом активов.

Таким образом, отказ от условия NA приводит к более простым и общим результатам. Следует отметить, что допустимость и даже желательность такого подхода пропагандируется в работах [5], [6], [13] и обусловлена следующими причинами (здесь имеется ввиду, что множество $J$ конечно). Во-первых, условие NUPBR (в отличие от NA) инвариантно относительно замены дисконтирующего актива (выбора единицы измерения). Во-вторых, именно условие NUPBR допускает "конструктивное" описание в терминах триплета предсказуемых характеристик семимартингала $S$. В третьих, данное условие эквивалентно условию существования эталонного портфеля (numéraire portfolio), т.е. такого положительного процесса $V \in \mathbb{W}(\mathbb{S})$, что $X / V$ является Р-супермартингалом для любого $X \in \mathbb{W}(\mathbb{S})$. В четвертых, только условие NUPBR существенно при рассмотрении задач оптимального инвестирования и т.п. 
2. Доказательства. Прежде всего напомним два известных результата: принцип компактности в $L_{+}^{0}[11 ;$ лемма A1.1] и теорему о седловой точке (см., например, [14; теорема 2.10.2]).

Лемма 1. Пусть $f_{n} \in L_{+}^{0}, n \geqslant 1, u$ множество $\operatorname{conv}\left(f_{n} ; n \geqslant 1\right)$ ограничено по вероятности. Тогда существует последовательность $g_{n} \in \operatorname{conv}\left(f_{m} ; m \geqslant n\right)$, сходящаяся п.н. к функиии $g<\infty$.

Лемма 2. Пусть $A, B$ - выпуклье компактные подмножества локально выпукльх векторных пространств $X, Y$ соответственно, и пусть отображение $\Phi: A \times B \mapsto \mathbb{R}$ обладает следующими свойствами:

(i) при каждом $b \in B$ функиия $a \mapsto \Phi(a, b)$ вогнута и полунепрерьвна сверху;

(ii) при каждом $a \in A$ бункция $b \mapsto \Phi(a, b)$ выпукла и полунепрерывна снизу.

Тогда $\Phi$ обладает седловой точкой, т.е. существует пара $(\bar{a}, \bar{b}) \in A \times B$ такая, что

$$
\Phi(a, \bar{b}) \leqslant \Phi(\bar{a}, \bar{b}) \leqslant \Phi(\bar{a}, b), \quad(a, b) \in A \times B .
$$

Идея следующей леммы заимствована из работы [5]. Рассуждения [5], следуя которым можно провести ее доказательство, опираются на результаты [3]. Ниже дается другое, более непосредственное доказательство, основанное на леммах 1 и 2. Напомним, что множество $F \subset L_{+}^{0}$ называется телесным, если из условий $f \in F$, $0 \leqslant g \leqslant f$ вытекает, что $g \in F$.

Лемма 3. Пусть множество $F \subset L_{+}^{0}$ содержит 1 и является выпуклым, телесным, ограниченным и замкнутым в $L^{0}$. Тогда существует единственный элемент $v \in F, v>0$, такой, что

$$
\mathrm{E}\left(\frac{u}{v}\right) \leqslant 1, \quad u \in F
$$

Доказательство. Рассмотрим множество $F_{N}=\{x \wedge N: x \in F\}$. В силу телесности $F$ имеем $F_{N} \subset F$. Из вогнутости функции $a \mapsto a \wedge N$, а также выпуклости и телесности $F$ очевидным образом вытекает, что $F_{N}$ выпукло.

Покажем, что $F_{N}$ замкнуто в $L^{0}$. Пусть последовательность $y_{n} \in F_{N}$ сходится к $y$ по вероятности. Переходя при необходимости к подпоследовательности, без ограничения общности можно считать, что $y_{n}=x_{n} \wedge N \rightarrow y$ п.н., где $x_{n} \in F$. По лемме 1 при каждом $n$ существует последовательность неотрицательных чисел $\left(\lambda_{j}^{n}\right)_{j=n}^{\infty}$, лишь конечное число элементов которой отличны от нуля, такая, что

$$
\widetilde{x}_{n}=\sum_{j=n}^{\infty} \lambda_{j}^{n} x_{j} \rightarrow x \text { п.н., } \quad \sum_{j=n}^{\infty} \lambda_{j}^{n}=1 .
$$

При этом $x<\infty$, так как $F$ ограничено в $L^{0}$, и $x \in F$ в силу замкнутости $F$ в $L^{0}$. Ясно, что последовательность $\widetilde{y}_{n}=\sum_{j=n}^{\infty} \lambda_{j}^{n} y_{j}$ сходится к $y$ п.н. Но

$$
\widetilde{y}_{n}=\sum_{j=n}^{\infty} \lambda_{j}^{n}\left(x_{j} \wedge N\right) \leqslant\left(\sum_{j=n}^{\infty} \lambda_{j}^{n} x_{j}\right) \wedge N .
$$

Следовательно, $y \leqslant x \wedge N$ и $y \in F$. 
Пусть $L^{1}=L^{1}(\Omega, \mathscr{F}, \mathrm{P})$ и $L^{\infty}=L^{\infty}(\Omega, \mathscr{F}, \mathrm{P})$ - пространства интегрируемых и существенно ограниченных функций соответственно. Множество $F_{N}$ является компактным в $*$-слабой топологии $\sigma\left(L^{\infty}, L^{1}\right)$ пространства $L^{\infty}$. Для обоснования данного утверждения достаточно установить, что $F_{N}$ замкнуто в топологии Макки $\tau\left(L^{\infty}, L^{1}\right)$, и воспользоваться теоремами Макки-Аренса и Банаха-Алаоглу. Заметим, что топология нормы на пространстве $L^{1}$ является топологией равномерной сходимости на шаре

$$
\left\{x \in L^{\infty}:\|x\|_{\infty}=\operatorname{ess} \sup |x| \leqslant 1\right\},
$$

который как подмножество $L^{1}$ является $\sigma\left(L^{1}, L^{\infty}\right)$-компактным. Поэтому сужение топологии нормы $L^{1}$ на $L^{\infty}$ слабее топологии Макки $\tau\left(L^{\infty}, L^{1}\right)$. Выше установлено, что множество $F_{N} \subset\left\{x \in L^{\infty}:\|x\|_{\infty} \leqslant N\right\}$ замкнуто в $L^{0}$. Следовательно, оно замкнуто в $L^{1}$, а значит, и в топологии $\tau\left(L^{\infty}, L^{1}\right)$.

Рассмотрим функцию $\Phi_{N}: F_{N} \times F_{N} \rightarrow \mathbb{R}$ вида

$$
\Phi_{N}(x, y)=\mathrm{E}\left(x \psi_{N}(y)\right), \quad \psi_{N}(a)=\frac{1}{a} I_{[1 / N, \infty)}(a)+\left(N+N^{2}\left(\frac{1}{N}-a\right)\right) I_{[0,1 / N)}(a) .
$$

Легко видеть, что функция $\psi_{N}:[0, \infty) \rightarrow[0, \infty)$ является ограниченной, невозрастающей, выпуклой и непрерывно дифференцируемой. Кроме того, функция $N \mapsto \psi_{N}(a)$ при каждом $а$ является неубывающей на $(0, \infty)$ и $\psi_{N}(a)=1 / a$ при $N \geqslant 1 / a$.

Очевидно, что функция $\Phi_{N}(\cdot, y)$ линейна и $\sigma\left(L^{\infty}, L^{1}\right)$-непрерывна на $F_{N}$ при всех $y \in F_{N}$, а функция $\Phi_{N}(x, \cdot)$ выпукла при всех $x \in F_{N}$. Покажем, что $\Phi_{N}(x, \cdot)$ полунепрерывна снизу в топологии $\sigma\left(L^{\infty}, L^{1}\right)$, т.е. что множество

$$
A_{N}=\left\{y \in F_{N}: \mathrm{E}\left(x \psi_{N}(y)\right) \leqslant c\right\}
$$

замкнуто в $\sigma\left(L^{\infty}, L^{1}\right)$ при всех $c \in \mathbb{R}$.

Пусть у принадлежит $\sigma\left(L^{\infty}, L^{1}\right)$-замыканию $A_{N}$. Поскольку топология $\sigma\left(L^{\infty}, L^{1}\right)$ сильнее топологии $\sigma\left(L^{1}, L^{\infty}\right)$ на $L^{\infty}$, то $y$ принадлежит $\sigma\left(L^{1}, L^{\infty}\right)$-замыканию $A_{N}$. Но топология нормы на $L^{1}$ согласована с двойственностью $\left\langle L^{1}, L^{\infty}\right\rangle$, и замыкание выпуклого множества $A_{N}$ в указанной топологии совпадает с его $\sigma\left(L^{1}, L^{\infty}\right)$-замыканием. Пусть $y_{n} \in F_{N}$ - последовательность, сходящаяся к $y$ в топологии нормы $L^{1}$ (а значит, и по вероятности). Тогда

$$
\mathrm{E}\left(x \psi_{N}(y)\right)=\lim _{n \rightarrow \infty} \mathrm{E}\left(x \psi_{N}\left(y_{n}\right)\right) \leqslant c
$$

по теореме о мажорируемой сходимости. Следовательно, $y \in A_{N}$.

По лемме 2 функция $\Phi_{N}$ обладает седловой точкой $\left(x_{N}, y_{N}\right)$ на $F_{N} \times F_{N}$ :

$$
\Phi_{N}\left(x, y_{N}\right) \leqslant \Phi_{N}\left(x_{N}, y_{N}\right) \leqslant \Phi_{N}\left(x_{N}, y\right), \quad(x, y) \in F_{N} \times F_{N} .
$$

Поскольку $\psi_{N}(a) \leqslant 1 / a, N>0$, то, полагая $y=x_{N}$, получаем неравенство

$$
\Phi_{N}\left(x, y_{N}\right) \leqslant \mathrm{E}\left(x_{N} \psi_{N}\left(x_{N}\right)\right) \leqslant 1, \quad x \in F_{N} .
$$

По лемме 1 существует последовательность

$$
v_{N}=\sum_{j \geqslant N} \lambda_{j}^{N} y_{j} \rightarrow v, \quad N \rightarrow \infty
$$


Здесь $\lambda_{j}^{N} \geqslant 0$ и лишь конечное число элементов последовательности $\left(\lambda_{j}^{N}\right)_{j=N}^{\infty}$ отличны от нуля. Из выпуклости, ограниченности и замкнутости $F$ в $L^{0}$ вытекает, что $v<\infty, v \in F$. Покажем, что $v>0$ п.н.

В силу выпуклости функции $a \mapsto \psi_{N}(a)$ и монотонности функции $N \mapsto \psi_{N}(a)$ имеем

$$
\Phi_{N}\left(x, v_{N}\right) \leqslant \sum_{j \geqslant N} \lambda_{j}^{N} \mathrm{E}\left(x \psi_{N}\left(y_{j}\right)\right) \leqslant \sum_{j \geqslant N} \lambda_{j}^{N} \mathrm{E}\left(x \psi_{j}\left(y_{j}\right)\right) \leqslant 1
$$

где $x \in F_{N}$. Пусть $\alpha \in(0,1)$ и $N \geqslant 1 / \alpha$. Тогда

$$
1 \geqslant \Phi_{N}\left(1, v_{N}\right) \geqslant \mathrm{E}\left(\psi_{N}\left(v_{N}\right) I_{\left\{v_{N} \leqslant \alpha\right\}}\right) \geqslant \psi_{N}(\alpha) \mathrm{P}\left(v_{N} \leqslant \alpha\right)=\frac{\mathrm{P}\left(v_{N} \leqslant \alpha\right)}{\alpha} .
$$

Далее, используя неравенство

$$
I_{\{v=0\}} \leqslant \lim _{N \rightarrow \infty} \inf _{m \geqslant N} I_{\left\{v_{m} \leqslant \alpha\right\}}
$$

и лемму Фату, находим

$$
\mathrm{P}(v=0) \leqslant \mathrm{E}\left(\liminf _{N \rightarrow \infty} I_{\left\{v_{N} \leqslant \alpha\right\}}\right) \leqslant \liminf _{N \rightarrow \infty} \mathrm{P}\left(v_{N} \leqslant \alpha\right) \leqslant \alpha .
$$

В силу произвольности $\alpha \in(0,1)$ это означает, что $\mathrm{P}(v=0)=0$.

Таким образом, $\mathrm{E}\left(x \psi_{N}\left(v_{N}\right)\right) \leqslant 1, x \in F_{N}$ и $v_{N} \rightarrow v, 0<v<\infty$ п.н. Учитывая, что $\psi_{N}\left(v_{N}\right) \rightarrow 1 / v$ п.н., по лемме Фату заключаем, что $\mathrm{E}(x / v) \leqslant 1, x \in F_{M}$, для любого $M>0$. Для произвольного $u \in F$ имеем $u \wedge M \in F_{M}$, и неравенство (2.1) вытекает из теоремы о монотонной сходимости при $M \uparrow \infty$.

Установим единственность $v$. Если $w \in F-$ положительный элемент, удовлетворяющий условию $\mathrm{E}(u / w) \leqslant 1, u \in F$, и $\mathrm{P}(v \neq w)>0$, то, применяя неравенство Йенсена в строгой форме, получаем противоречие:

$$
1 \geqslant \mathrm{E} \frac{w}{v}>\frac{1}{\mathrm{E}(v / w)} .
$$

Лемма доказана.

Отметим, что теорема о минимаксе для функций, заданных на подмножествах $L_{+}^{0}$, получена в работе [15]. Однако лемма 3 не является прямым следствием результатов [15].

ДОКАЗАТЕЛЬСТВО ТЕОРЕМЫ 1 . (i) $\Rightarrow$ (ii). При любом $t \geqslant 0$ множество

$$
H_{t}=\left\{y \in L_{+}^{0}\left(\Omega, \mathscr{F}_{t}, \mathrm{P}\right): y \leqslant X_{t} \text { для некоторого } X \in \mathbb{W}\right\} \subset H
$$

выпукло, телесно, ограничено по вероятности и содержит 1. Ясно, что замыкание $\mathrm{cl}_{0}\left(H_{t}\right)$ множества $H_{t}$ в $L^{0}$ удовлетворяет условиям леммы 3 . Следовательно, существует согласованный с фильтрацией случайный процесс $V, 0<V_{t} \in \operatorname{cl}_{0}\left(H_{t}\right)$, такой, что

$$
\mathrm{E}\left(\frac{X_{t}}{V_{t}}\right) \leqslant 1, \quad X \in \mathbb{W} .
$$

Пусть последовательность $y^{k} \in H_{u}$ сходится к $V_{u}$ в $L^{0}$. Не ограничивая общности, можно считать, что имеет место и сходимость с вероятностью 1. Рассмотрим 
последовательность $X^{k} \in \mathbb{W}: y^{k} \leqslant X_{u}^{k}$. Применив дважды лемму 1 , построим последовательность $Y^{k} \in \mathbb{W}, Y^{k} \in \operatorname{conv}\left(X^{m} ; m \geqslant k\right)$, такую, что $Y_{t}^{k} \rightarrow W_{t}$ п.н., $k \rightarrow \infty$, при $t \in\{s, u\}, 0 \leqslant s<u$. Покажем, что $W_{t}=V_{t}$, при $t \in\{s, u\}$. Подчеркнем, что здесь не утверждается существование предельного процесса $W$ при $t \notin\{s, u\}$.

Ясно, что

$$
W_{u} \geqslant \lim _{k \rightarrow \infty} y^{k}=V_{u} .
$$

Из неравенства (2.2) и леммы Фату следует, что $W_{u}=V_{u}$ п.н.

Далее, построим последовательность

$$
\widehat{Y}^{k} \in \mathbb{W}, \quad \widehat{Y}_{s}^{k} \rightarrow V_{s} \text { п.н., } \quad k \rightarrow \infty,
$$

и последовательность $V^{k} \in \mathbb{W}$ вида

$$
V_{t}^{k}=\widehat{Y}_{t}^{k} I_{[0, s)}(t)+\frac{\alpha^{k} Y_{t}^{k}+1-\alpha^{k}}{\alpha^{k} Y_{s}^{k}+1-\alpha^{k}} \widehat{Y}_{s}^{k} I_{[s, \infty)}(t),
$$

где $\alpha^{k} \in(0,1)$. При $\alpha^{k} \rightarrow 1$ имеем

$$
\frac{V_{u}^{k}}{V_{u}}=\frac{\alpha^{k} Y_{u}^{k}+1-\alpha^{k}}{V_{u}} \frac{\widehat{Y}_{s}^{k}}{\alpha^{k} Y_{s}^{k}+1-\alpha^{k}} \rightarrow \frac{V_{s}}{W_{s}} \text { п.н. }
$$

Согласно лемме Фату и неравенству (2.2)

$$
\mathrm{E} \frac{V_{s}}{W_{s}} \leqslant \liminf _{k \rightarrow \infty} \mathrm{E}\left(\frac{V_{u}^{k}}{V_{u}}\right) \leqslant 1 .
$$

С другой стороны, по тем же соображениям

$$
\mathrm{E} \frac{W_{s}}{V_{s}} \leqslant \liminf _{k \rightarrow \infty} \mathrm{E} \frac{Y_{s}^{k}}{V_{s}} \leqslant 1 .
$$

Отсюда следует, что $V_{s} / W_{s}=1$ п.н. Действительно, если случайная величина $V_{s} / W_{s}$ не равна константе п.н., то, воспользовавшись неравенством Йенсена в строгой форме, получаем противоречие:

$$
\mathrm{E} \frac{V_{s}}{W_{s}}>\left(\mathrm{E} \frac{W_{s}}{V_{s}}\right)^{-1} \geqslant 1
$$

Итак,

$$
Y_{t}^{k} \rightarrow V_{t} \text { п.н., } \quad k \rightarrow \infty, \quad \text { при } t \in\{s, u\} .
$$

Покажем, что для любого $X \in \mathbb{W}$ процесс $X / V$ является супермартингалом (идея следующего рассуждения заимствована из работы [16]). Пусть это неверно. Тогда существует $X \in \mathbb{W}$ и неотрицательные числа $s<u$ такие, что вероятность события

$$
A=\left\{\omega: \mathrm{E}\left(\frac{X_{u}}{V_{u}} \mid \mathscr{F}_{s}\right)>\frac{X_{s}}{V_{s}}\right\} \in \mathscr{F}_{s}
$$

положительна. 
Не ограничивая общности, можно считать, что $X_{s}>0$ на $A$. Действительно, пусть $X_{s}=0$ на множестве $B \in \mathscr{F}_{s}$. Положим

$$
\widehat{X}_{t}^{k}=I_{[0, s)}(t)+\left(I_{B} \frac{1-\alpha^{k}+\alpha^{k} X_{t}}{1-\alpha^{k}+\alpha^{k} X_{s}}+I_{B^{c}}\right) I_{[s, \infty)}(t),
$$

где $\alpha^{k} \in(0,1), \alpha^{k} \rightarrow 1$. Поскольку $\widehat{X}^{k} \in \mathbb{W}$, то множество $H$ содержит последовательность

$$
\widehat{X}_{u}^{k}=I_{B} \frac{1-\alpha^{k}+\alpha^{k} X_{u}}{1-\alpha^{k}}+I_{B^{c}} .
$$

Из ограниченности $H$ в $L^{0}$ вытекает, что $X_{u}=0$ п.н. на $B$.

Введем последовательность процессов $U^{k} \in \mathbb{W}$ по формуле

$$
U_{t}^{k}=Y_{t}^{k} I_{[0, s)}(t)+Y_{s}^{k}\left(I_{A} \frac{\alpha^{k} X_{t}+1-\alpha^{k}}{\alpha^{k} X_{s}+1-\alpha^{k}}+I_{A^{c}} \frac{\alpha^{k} Y_{t}^{k}+1-\alpha^{k}}{\alpha^{k} Y_{s}^{k}+1-\alpha^{k}}\right) I_{[s, \infty)}(t),
$$

где $\alpha^{k} \in(0,1)$. Полагая $t=u$ и переходя к пределу при $\alpha^{k} \rightarrow 1$, находим

$$
U_{u}=\lim _{k \rightarrow \infty} U_{u}^{k}=I_{A} X_{u} \frac{V_{s}}{X_{s}}+V_{u} I_{A^{c}} \text { п.н. }
$$

Наконец, используя неравенство (2.2), получаем противоречие:

$$
\begin{aligned}
1 \geqslant \liminf _{k \rightarrow \infty} \mathrm{E} \frac{U_{u}^{k}}{V_{u}} & \geqslant \mathrm{E}\left(I_{A} \frac{X_{u}}{V_{u}} \frac{V_{s}}{X_{s}}+I_{A^{c}}\right) \\
& =\mathrm{E}\left(I_{A} \frac{V_{s}}{X_{s}} \mathrm{E}\left(\frac{X_{u}}{V_{u}} \mid \mathscr{F}_{s}\right)\right)+\mathrm{P}\left(A^{c}\right)>\mathrm{P}(A)+\mathrm{P}\left(A^{c}\right)=1 .
\end{aligned}
$$

Супермартингал $1 / V$ может не быть элементом $\mathbb{D}$. Поэтому рассмотрим его регуляризацию:

$$
Z_{t}=\liminf _{s \downarrow t, s \in \mathbb{Q}} \frac{1}{V_{s}}
$$

где $\mathbb{Q}$ - множество рациональных чисел. Теорема Дуба о регуляризации [17; теорема 6.27] показывает, что

$$
Z_{t}=\lim _{s \downarrow t, s \in \mathbb{Q}} \frac{1}{V_{s}} \text { п.н., }
$$

$Z$ принадлежит $\mathbb{D}$ и является супермартингалом (точнее, траектории $Z$ совпадают с траекториями такого процесса с вероятностью 1).

Процесс $Z$ является супермартингальной плотностью для $\mathbb{W}$. Действительно, пусть $u<v$ и $u_{n} \downarrow u, v_{n} \downarrow v, u_{n}, v_{n} \in \mathbb{Q}$. Тогда

$$
\begin{aligned}
& \mathrm{E}\left(X_{v} Z_{v} \mid \mathscr{F}_{s}\right) \leqslant \liminf _{n \rightarrow \infty} \mathrm{E}\left(\frac{X_{v_{n}}}{V_{v_{n}}} \mid \mathscr{F}_{s}\right) \leqslant \frac{X_{s}}{V_{s}}, \quad s<v, \\
& \mathrm{E}\left(X_{v} Z_{v} \mid \mathscr{F}_{u}\right)=\lim _{n \rightarrow \infty} \mathrm{E}\left(X_{v} Z_{v} \mid \mathscr{F}_{u_{n}}\right) \leqslant \lim _{n \rightarrow \infty} \frac{X_{u_{n}}}{V_{u_{n}}}=X_{u} Z_{u} .
\end{aligned}
$$

Остается проверить, что $Z_{\infty}>0$ п.н. Согласно предложению 2.3 (а) работы [18] равенство $Z_{t}=1 / V_{t}$ п.н. имеет место при всех $t \in \mathbb{R}_{+} \backslash K$, где $K$ - некоторое счетное множество. Множество

$$
\left\{\frac{1}{Z_{t}}: t \in \mathbb{R}_{+} \backslash K\right\} \subset\left\{V_{t}: t \geqslant 0\right\} \subset \bigcup_{t \geqslant 0} \operatorname{cl}_{0}\left(H_{t}\right) \subset \operatorname{cl}_{0}(H)
$$

ограничено в $L^{0}$. Отсюда следует, что $Z_{\infty}>0$ п.н. 
(ii) $\Rightarrow$ (i). Пусть $Z$ - эквивалентная супермартингальная плотность для $\mathbb{W}$. Пусть $y \in H$ и $X_{T}=X_{\infty}^{T} \geqslant y, X \in \mathbb{W}$. Тогда

$$
\mathrm{P}\left(y Z_{\infty} \geqslant A\right) \leqslant \mathrm{P}\left(X_{\infty}^{T} Z_{\infty} \geqslant A\right) \leqslant \frac{\mathrm{E}\left(X_{\infty}^{T} Z_{\infty}\right)}{A} \leqslant \frac{1}{A} .
$$

Следовательно, множество $Z_{\infty} H$ ограничено в $L^{0}$. Но оператор умножения на $Z_{\infty}^{-1}$ непрерывен в $L^{0}$. Поэтому $H$ также ограничено в $L^{0}$. Теорема доказана.

ДокАЗАтЕЛЬСтво теоремы 2. Достаточно проверить, что множество (1.3) удовлетворяет условиям теоремы 1 . Покажем, что $\mathbb{W}(\mathbb{S})$ является разветвленно-выпуклым (ясно, что остальные условия теоремы 1 выполнены). Пусть

$$
X^{i} \in \mathbb{W}(\mathbb{S}), \quad i=1,2,3, \quad X^{2}>0, \quad X^{3}>0
$$

и $h_{s} \in L_{+}^{0}\left(\mathscr{F}_{s}\right), h_{s} \leqslant 1$. Из определения множества $\mathbb{W}(\mathbb{S})$ следует, что процессы $X^{i}$ допускают представление $X^{i}=1+\theta^{i} \circ S, i=1,2,3$, где $\theta^{i} \circ S$ - стохастические интегралы по фиксированному семимартингалу $S$ с конечным числом компонент. Покажем, что аналогичное представление имеет место и для процесса вида (1.1). Положим

$$
\theta_{t}=\theta_{t}^{1} I_{[0, s]}(t)+\left(\theta_{t}^{2} \frac{X_{s}^{1}}{X_{s}^{2}} h_{s}+\theta_{t}^{3} \frac{X_{s}^{1}}{X_{s}^{3}}\left(1-h_{s}\right)\right) I_{(s, \infty)}(t) .
$$

Тогда

$$
\begin{aligned}
X_{t}=1+(\theta \circ S)_{t}=1 & +\left(\theta^{1} \circ S\right)_{t \wedge s}+\frac{X_{s}^{1}}{X_{s}^{2}} h_{s}\left(\left(\theta^{2} \circ S\right)_{t}-\left(\theta^{2} \circ S\right)_{t \wedge s}\right) \\
& \quad+\frac{X_{s}^{1}}{X_{s}^{3}}\left(1-h_{s}\right)\left(\left(\theta^{3} \circ S\right)_{t}-\left(\theta^{3} \circ S\right)_{t \wedge s}\right) \\
= & X_{t \wedge s}^{1}+\frac{X_{s}^{1}}{X_{s}^{2}} h_{s}\left(X_{t}^{2}-X_{t \wedge s}^{2}\right)+\frac{X_{s}^{1}}{X_{s}^{3}}\left(1-h_{s}\right)\left(X_{t}^{3}-X_{t \wedge s}^{3}\right) \\
= & X_{t}^{1} I_{[0, s)}(t)+\left(h_{s} \frac{X_{s}^{1}}{X_{s}^{2}} X_{t}^{2}+\left(1-h_{s}\right) \frac{X_{s}^{1}}{X_{s}^{3}} X_{t}^{3}\right) I_{(s, \infty)}(t) .
\end{aligned}
$$

\section{СПИСОК ЦИТИРОВАННОЙ ЛИТЕРАТУРЫ}

[1] J. Jacod, A. N. Shiryaev, Limit Theorems for Stochastic Processes, Grundlehren Math. Wiss., 288, Springer-Verlag, Berlin, 2003.

[2] G. Žitković, "A filtered version of the bipolar theorem of Brannath and Schachermayer", J. Theoret. Probab., 15:1, (2002), 41-61.

[3] D. Kramkov, W. Schachermayer, "The asymptotic elasticity of utility functions and optimal investment in incomplete markets", Ann. Appl. Probab., 9:3, (1999), 904-950.

[4] Yu. Kabanov, C. Stricker, "Remarks on the true no-arbitrage property", Séminaire de Probabilités XXXVIII, Lecture Notes in Math., 1857, Springer-Verlag, Berlin, 2005, 186-194.

[5] M. M. Christensen, K. Larsen, "No arbitrage and the growth optimal portfolio", Stoch. Anal. Appl., 25:1, (2007), 255-280.

[6] I. Karatzas, C. Kardaras, "The numéraire portfolio in semimartingale financial models", Finance Stoch., 11:4, (2007), 447-493. 
[7] F. Delbaen, W. Schachermayer, The Mathematics of Arbitrage, Springer Finance, Springer-Verlag, Berlin, 2006.

[8] Ю. М. Кабанов, Д. О. Крамков, "Большие финансовые рынки: асимптотический арбитраж и контигуальность", ТВП, 39:1, (1994), 222-229.

[9] Yu. M. Kabanov, D. O. Kramkov, "Asymptotic arbitrage in large financial markets", Finance Stoch., 2:2, (1998), 143-172.

[10] M. De Donno, P. Guasoni, M. Pratelli, "Super-replication and utility maximization in large financial markets", Stochastic Process. Appl., 115:12, (2005), 2006-2022.

[11] F. Delbaen, W. Schachermayer, "A general version of the fundamental theorem of asset pricing", Math. Ann., 300:3, (1994), 463-520.

[12] F. Delbaen, W. Schachermayer, "The fundamental theorem of asset pricing for unbounded stochastic processes", Math. Ann., 312:2, (1998), 215-250.

[13] E. Platen, D. Heath, A Benchmark Approach to Quantitative Finance, Springer Finance, Springer-Verlag, Berlin, 2006.

[14] C. Zălinescu, Convex Analysis in General Vector Spaces, World Sci. Publ., Singapore, 2002.

[15] M. Pratelli, "A minimax theorem without compactness hypothesis", Mediterr. J. Math., 2:1, (2005), 103-112.

[16] D. Becherer, "The numéraire portfolio for unbounded semimartingales", Finance Stoch., 5:3, (2001), 327-341.

[17] O. Kallenberg, Foundations of Modern Probability, Probab. Appl. (N. Y.), Springer-Verlag, New York, NY, 1997.

[18] I. Karatzas, G. Žitković, "Optimal consumption from investment and random endowment in incomplete semimartingale markets", Ann. Probab., 31:4, (2003), 1821-1858.

Д. Б. Рохлин

Южный федеральный университет, г. Ростов-на-Дону

E-mail: rokhlin@math.rsu.ru
Поступило

04.06.2007

Исправленный вариант

15.08.2009 